\title{
Ärztliche Identität jenseits von Idealisierung und Anpassung an den Zeitgeist - gibt es das ?*
}

Paul Hoff

Chefarzt und stv. Klinikdirektor, Psychiatrische Universitätsklinik Zürich

* Überarbeitete Fassung eines Vortrages an den 2. Zürcher Gesundheitstagen, veranstaltet von der Ärztegesellschaft des Kantons Zürich AGZ (28./29. März 2008).

** Berufs- und Funktionsbezeichnungen werden aus Gründen der Lesbarkeit nur in ihrer männlichen Form gebraucht.

Korrespondenz:

Prof. Dr. med. Dr. phil. Paul Hoff Psychiatrische Universitätsklinik Zürich

Klinik für soziale Psychiatrie und Allgemeinpsychiatrie ZH West Lenggstrasse 31

Postfach 1931

CH-8032 Zürich

paul.hoff@puk.zh.ch

\section{Einleitung}

Einige der Begriffe, die im Titel dieses Beitrags erscheinen, wirken schnell als Schlagworte. Um eine solche Art der Auseinandersetzung mit dem Thema zu vermeiden, soll zunächst die Fragestellung präzisiert werden. Im Anschluss wird begründet, warum gerade die Psychiatrie für die hier angeschnittenen Themen als pars pro toto für die Medizin stehen kann. Schliesslich werden unterschiedliche, wenn auch eng miteinander verknüpfte Dimensionen ärztlicher Identität beschrieben und auf ihre Praxisrelevanz hin untersucht. Ein Resümee in vier Thesen schliesst den Beitrag ab.

\section{Präzisierung der Fragestellung}

Zunächst sollen die beiden Extreme eines naiv idealisierenden, gleichsam «absolutistischen» Arztbildes** $^{*}$ und einer nicht minder naiven, «relativistischen» Anpassung an den jeweiligen Zeitgeist einander gegenübergestellt werden.

Ein naiv idealisierendes Arztbild geht typischerweise von einem unreflektierten Paternalismus aus: Dem Arzt wird die nahezu unbeschränkte Autorität für Entscheidungen zugewiesen, die das Gesamtfeld der - auch langfristigen Untersuchung und Behandlung des Patienten betreffen. Ein solches Arztbild, wie es über viele Jahre nicht nur praktiziert, sondern auch gelehrt wurde, gerät vor allem in Zeiten schnellen gesellschaftlichen Wandels rasch in eine «splendid isolation»: Eine Zeitlang genügt es sich selbst und kümmert sich wenig um die Vernetzung mit wissenschaftlichen Nachbardisziplinen und mit gesellschaftlich wichtigen Kräften. Ein solches ärztliches Selbstverständnis wird freilich in jüngerer Zeit von der Gesellschaft allgemein und von den einzelnen Patienten nicht mehr einfach akzeptiert: Arzt und Ärztin sind nicht qua Amt uneingeschränkte Autoritäten, ihre Rollen in Medizin und Gesellschaft werden kritisch hinterfragt. Nachbarberufe und -wissenschaften erheben Ansprüche auf ehemals medizinische Tätigkeitsbereiche. In dieser Situation ist die naive Idealisierung sicher keine gute Position für die Ärzteschaft. Denn sie regt kaum zu kritischer Reflexion über grundlegende, aber eben auch praxisrelevante Fragen an, etwa denjenigen nach dem Verständnis von Krankheit und Gesundheit, nach dem sinnvollsten Wissenschaftsverständnis

\section{Existe-t-il une identité médicale au-delà d'une idéalisation \\ naïve et d'une adaptation à}

\section{I'esprit du temps?}

L'«identité médicale» n'est-elle plus qu'un cliché souvent utilisé, mais rarement rempli d'un contenu concret? D'un point de vue objectif, cette identité demeure, certes, le point de référence nécessaire de chaque acte médical, aujourd'hui encore. Par contre, toute idéalisation naïve de l'image du médecin ou son adaptation à l'esprit du temps sont dommageables. L'identité médicale doit maintenant remplir les exigences du $21^{\mathrm{e}}$ siècle et adopter une forme répondant aux besoins du temps et des patients tout en renforçant la confiance en soi de la profession. Cette question ne doit pas seulement être examinée par les sociologues et les politiciens de la santé, mais aussi être activement débattue au sein du corps médical. L'élément central de chaque acte médical demeure la relation médecin-patient. Malgré tout le respect dû à l'importance indubitable des conditions économiques et sociétales, force est de constater qu'en subordonnant cette relation à la primauté de l'efficacité des prestations ou en la réduisant au seul accomplissement d'une demande dans le cadre de la médecine «Lifestyle», on est loin d'agir en faveur d'une identité médicale cohérente, efficace et crédible. Comme la psychiatrie dépend particulièrement $d$ 'une réflexion continue et critique sur ses présupposés théoriques, on est en droit, dans le présent contexte, de la désigner pour une fois comme pars pro toto pour toute la médecine.

für die Medizin und nach den zukünftigen Rollen von Arzt und Patient. Aber ohne eine medizininterne Debatte über genau diese Fragen wird sich eine brüchiger werdende ärztliche Identität nicht nachhaltig stabilisieren lassen. 
Dem gegenüber steht die andere Extremvariante einer naiven Anpassung der ärztlichen Identität an den jeweiligen Zeitgeist. Ein aktuelles Beispiel ist die Überakzentuierung der (vermeintlichen) Patientenautonomie im Sinne eines völlig verkürzten «shared clinical decision making», was nichts anderes ist als die bequeme, aber unärztliche Delegation von Verantwortung an den Patienten. Dieser wird verstanden als Empfänger von Informationen, die er selbst zu bewerten und zur Grundlage seiner sodann dem Arzt mitzuteilenden Entscheidung zu machen habe. Nun ist die reale Interaktion zwischen Arzt und Patient zumeist markant anders, vor allem viel komplexer.

Ein weiteres Merkmal dieser Variante ist die Gleichsetzung von ärztlichem Handeln mit einer beliebigen, den Gesetzen des Marktes unterworfenen Dienstleistung. Schliesslich führt die Strategie der blossen Anpassung, eben weil sie so wenig eigene Substanz hat, schnell zum Gefühl der Bedrohung durch die zunehmende Vernetzung ärztlicher Themen und Kompetenzen mit anderen Berufsfeldern. Dies weist freilich Parallelen zur Position der naiven Idealisierung, auf, insofern auch dort wenig Kontakt zu Nachbardisziplinen bestand, jedoch aus einer scheinbaren Position der Stärke heraus; hier, bei der naiven Anpassung, passiert das Gleiche auf dem Hintergrund einer geradezu ängstlichen Verunsicherung.

\section{Die Psychiatrie als pars pro toto für die Medizin}

Das psychiatrische und psychotherapeutische Feld fungiert, was eine ganze Reihe von medizinischen Grundsatzfragen angeht, gleichsam wie ein Brennglas. Dies liegt an bestimmten Besonderheiten psychiatrischen Handelns im medizinisch-gesellschaftlichen Kontext (Abb. 1).

Enger als andere medizinische Fachdisziplinen sind Psychiatrie und Psychotherapie mit gesellschaftlichen und politischen Entwicklungen vernetzt. Aber auch innerhalb der Psychiatrie selbst gibt es bedeutend schärfere Kontroversen über grundsätzliche Fragen, als wir dies aus anderen medizinischen Bereichen kennen. Zu erwähnen sind beispielhaft die Schulenbildung etwa naturalistisch-neurowissenschaftlicher und heuristisch-psychodynamischer Ausrichtung sowie die fundamentale Kritik der «Antipsychiatrie» der 60er und 70er Jahre des 20. Jahrhunderts. Diese hatte immerhin behauptet, die wesentlichen Kernbegriffe psychiatrischen Handelns, vor allem der Krankheitsbegriff selbst, seien gar keine wissenschaftlichen Termini, sondern geschickt versteckte Instrumentalisierungen gesellschaftlicher Macht gegenüber Menschen mit grundsätzlich anderer Lebensauffassung und anderem Verhalten. Freilich gibt es auch in der nichtpsychiatrischen Medizin Kontroversen, man denke etwa an die vielen Debatten um die «Alternativmedizin». Dennoch erreichen diese Kontroversen, gesamthaft betrachtet, oft nicht den Grad an Brisanz und praktischer Relevanz im täglichen ärztlichen Handeln, wie dies im psychiatrischen Kontext auch heute noch häufig zu beobachten ist.

Vermeintlich «rein» theoretische Fragen, die eng mit philosophischen Vorannahmen, speziell mit dem Menschenbild, zusammenhängen etwa nach dem Zusammenhang von Gehirnfunktion und psychischem Erleben oder den Vor- und Nachteilen subjekt- bzw. objektorientierter Methoden in der praktischen Diagnostik und in der Forschung -, sind aus der Psychiatrie nicht wegzudenken, auch wenn sie mitunter im Alltag als Ballast erlebt werden.

Nun betreffen all diese Aspekte im Grunde jedes medizinische Handeln. Und weil die (wissenschaftliche) Psychiatrie in ihrer 200-jährigen Geschichte besonders markant, ja schmerzhaft mit diesen Kontroversen konfrontiert war (und ist), wird man sie mit Blick auf die ärztliche Identität durchaus als pars pro toto für die Medizin betrachten dürfen. Zugespitzt resultieren die folgenden Frage: Kann es bei den vielen heterogenen Konzepten, die sich auf wissenschaftliche Ergebnisse von Nachbardisziplinen, etwa den Neurowissenschaften, stützen, im 21. Jahrhundert überhaupt noch so etwas wie eine Identität der Psychiatrie geben? Ist Psychiatrie nicht längst eine obsolete Bezeichnung für angewandte Neurobiologie, Neuropsychologie und Sozialwissenschaft? Bezogen auf die gesamte Medizin: Kann es bei diesen heterogenen, oft aus Nachbardisziplinen stammenden Konzepten im 21. Jahrhundert überhaupt noch eine Identität der Medizin und damit des Arztes geben? Ist Medizin nicht viel mehr bereits heute eine weitgehend obsolete Bezeichnung für angewandte Molekularbiologie, Medizintechnik und Sozialwissenschaft?

Um die verblüffende Praxisrelevanz theoretischer Aspekte zu veranschaulichen, seien wiederum psychiatrische Beispiele vorgebracht, nämlich die verschiedenen Definitionen von psychischer Erkrankung. Sie sind eben nicht «nur» theoretische Konstrukte, sondern wirken sich direkt auf das ärztliche Handeln aus. Die vier wichtigsten Definitionen verstehen psychische Krankheit als

- neurobiologische Dysfunktion;

- lebensgeschichtlich verstehbare, individuelle Fehlentwicklung;

- gesellschaftlich verstehbare, im Individuum manifestierte Fehlentwicklung; 


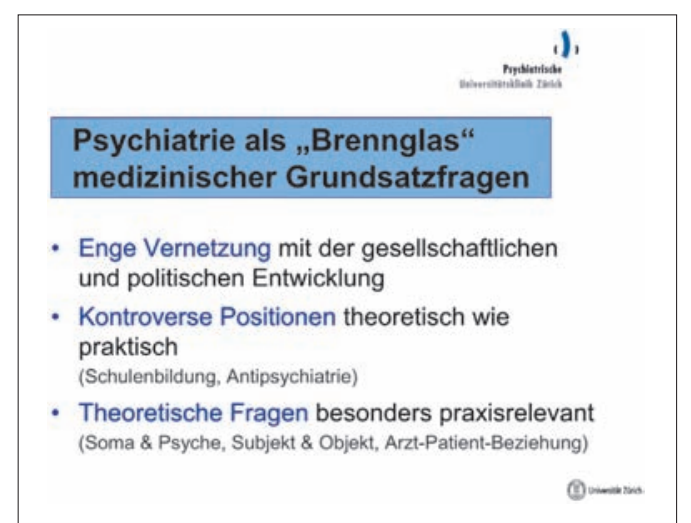

Abbildung 1

Psychiatrie als «Brennglas» medizinischer Grundsatzfragen.

- Bündel typischer beschreibbarer Symptome und Syndrome ohne ätiologische Vorannahmen (ICD10).

Die Überzeichnung einer dieser Perspektiven und eine solche ist durchaus nicht selten anzutreffen - hat nun erhebliche Folgen, auch für die ärztliche Identität Diese seien hier in pointierter Form erwähnt: Die unkritische Identifizierung des gesamten Phänomens psychische Störung mit neurobiologischer Dysfunktion, sei diese nun bereits bekannt oder bloss postuliert, macht den Arzt zum Techniker, der quantitative neurobiologische Veränderungen im ZNS des Patienten erkennt, diesem mitteilt und daraus Therapievorschläge ableitet. Überbetonte man hingegen den Aspekt des Verstehbaren an der psychischen Erkrankung, sei es individuell lebensgeschichtlich oder gesellschaftlich akzentuiert, so würde der Arzt, indem er andere relevante Dimensionen unterschätzt, zum Deuter oder Sozialberater. Im Falle des einseitigen Verständnisses psychischer Krankheit bloss als deskriptiv erfassbare Normabweichung - Prototyp: ICD10 - wird der Arzt zum psychiatrischen Informatiker, der isolierte psychopathologische Symptome mittels vordefinierter Algorithmen zu Diagnosen verknüpft.

Techniker, Deuter, Sozialberater, Informatiker: dies sind allesamt Begriffe, mit denen sich der in Klinik, Ambulanz oder Forschung tätige Psychiater sicher nicht identifizieren können wird. Und doch sind es durchaus realistische Konsequenzen einer unbedachten Überzeichnung theoretischer Konzepte, die das praktische ärztliche Tun leiten in der Psychiatrie wie andernorts.

\section{Dimensionen ärztlicher Identität und ihre Praxisrelevanz}

Ärztliche Identität im engeren Sinne hat zu tun mit Prävention, Erkennung und Behandlung von Erkrankungen. Dieser Kernbereich ist selbsterklärend. Im weiteren Sinne meint ärztliche Identität die aus den genannten Kernaktivitäten resultierende Verantwortung gegenüber der Gesellschaft, etwa im Hinblick auf die heute allgegenwärtige Kostenfrage, auf die Frage der Schwellenwerte für eine IV-Berentung oder auf die sehr spezielle Situation der forensischen Psychiatrie bei der Prognosebegutachtung. Schliesslich meint im weitesten und fachübergreifenden Sinne ärztliche Identität die Beteiligung an der gesellschaftlichen Debatte um unsere Vorstellung von Person und Bürger sowie um deren Weiterentwicklung, kurz die Frage des «Menschenbildes» (Abb. 2)

Die unmittelbare praktische Bedeutung der ärztlichen Identität im engeren Sinne zeigt sich am psychiatrischen Beispiel der oft schwierigen Grenzziehung zwischen psychischer Gesundheit und Krankheit: Sind Personen, die sich wegen leichter Verstimmungen beim Therapeuten melden, krank oder nicht krank? Sollen sie in diesem Verhalten aktiv gefördert und damit allenfalls in eine passive Patientenrolle manövriert werden, oder ist hier der Verweis auf Selbstwirksamkeit und Selbstverantwortung der angemessene ärztliche Rat? Und wie gehen wir mit psychisch gesunden Menschen um, die zur Verbesserung von Leistungsfähigkeit oder Allgemeinbefinden die Verordnung von Psychopharmaka wie Antidepressiva oder Stimulantien wünschen - Stichwort: «Lifestylepsychiatrie»?

Auch der - wiederum für uns Psychiater besonders prägnante - Spannungsbogen zwischen paternalistischer und autonomieorientierter Therapie belegt die Praxisrelevanz der Identitätsfrage: Ist es gerechtfertigt, gegenüber Personen mit psychischen Erkrankungen betont paternalistisch aufzutreten, in der selbstverständlichen Annahme, die Betroffenen selbst seien dazu krankheitsbedingt nicht in der Lage? Oder gibt es nicht sehr wohl den Anspruch des psychisch Kranken, trotz psychopathologischer Auffälligkeiten in seiner Autonomie, so weit irgend möglich, auch dann respektiert zu werden, wenn er etwa bei den geplanten Therapiemassnahmen zu einem ganz anderen Ergebnis kommt als der Therapeut?

Ärztliche Identität im weiteren Sinne konfrontiert uns mit der sehr praktischen Frage, was denn eigentlich das Therapieziel bei der psychiatrischen und psychotherapeutischen Behandlung ist: Ist es die Anpassung an die jeweilige Gesellschaft und deren Normen oder eher das Gegenteil, nämlich Kritik und Konfrontation? Diese Diskussion erinnert an heftige Debatten der «Antipsychiatrie» in den 60er und 70er Jahren des 20. Jahrhunderts, die der etablierten Psychiatrie ja vorwarf, mit wissenschaftlich fragwürdigen Begriffen wie Schizophrenie oder Persönlichkeitsstörung letztlich nur der Stabilisierung gesellschaftlicher Machtverhältnisse zu dienen 


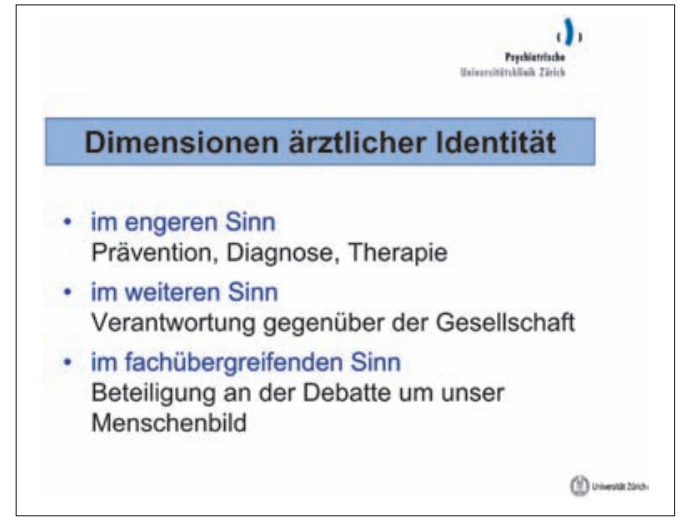

Abbildung 2

Dimensionen ärztlicher Identität.

und nicht das Wohl der Betroffenen im Auge zu haben. Hier sei an die soziogenetischen Modelle psychischer Störung erinnert, die davon ausgehen, dass ungünstige Lebensverhältnisse die spätere Entwicklung psychischer Erkrankungen mindestens fördern, möglicherweise sogar verursachen. Genau umgekehrt wurde aber auch die These vertreten, dass der soziale Abstieg, den psychisch Kranke häufig durchmachen, Folge der Erkrankung (und der gesellschaftlichen Reaktion auf diese) und nicht ihre Ursache ist.

Die Verantwortung gegenüber der Gesellschaft wird ebenfalls deutlich, wenn man sich die bereits erwähnte kontroverse Diskussion um den Anspruch psychisch Kranker auf Leistungen aus der Invalidenversicherung anschaut. Hier reicht die Spannbreite von der pauschalen Kritik an «der Psychiatrie», dass sie jedem, der dies wünsche, mit fragwürdigen Attesten und Bescheinigungen zu einer IV-Rente verhelfe, bis hin zur gegenteiligen Auffassung, dass sich wiederum «die Psychiatrie» von normativen Vorgaben wie der allgegenwärtigen Leistungsorientierung viel zu sehr und zu leichtfertig unter Druck setzen lasse, dies zu Lasten vor allem der schwer und chronisch Kranken. Ein weiteres brisantes Beispiel ist die Rolle der Psychiatrie im Umfeld der fürsorgerischen Freiheitsentziehung (FFE), die immer wieder, im Einzelfall wie generell, zu Kontroversen auch mit Blick auf das ärztliche Selbstverständnis führt.

Schliesslich ist die ärztliche Identität im fachübergreifenden Sinne gefragt, wenn es um übergeordnete Themen wie das Menschenbild im 21. Jahrhundert geht. Denn sie muss schliesslich wieder aus vorwiegend psychiatrischer Perspektive - Position beziehen zu den Fragen,

1. wie wir die rezenten Erkenntnisse der Neurowissenschaften mit dem tradierten Konzept der personalen Autonomie des Subjektes verbinden wollen (das klassische Problem der «Willensfreiheit»);
2. wie wir die Autonomie des psychisch kranken Subjektes feststellen und fördern, jedenfalls nicht unnötig einschränken wollen und

3. wie wir den Zusammenhang von Lebensgeschichte, Persönlichkeit und psychischer Erkrankung sowohl wissenschaftlich erfassen als auch therapeutisch nutzen können. Dies ist heute nicht Gegenstand des «mainstream», im Gegensatz zu den beiden Jahrzehnten nach dem 2. Weltkrieg, als zumindest im europäischen Umfeld die damals einflussreiche «anthropologische Psychiatrie» genau diesen Akzent gesetzt hatte.

\section{Ein Resümee in vier Thesen}

1. Dem Begriff «ärztliche Identität» droht zunehmend der Abstieg zu einem blossen Schlagwort, das zwar häufig verwandt, nur selten aber mit konkretem Inhalt gefüllt wird. Dabei ist diese Identität notwendiger Bezugspunkt jeden ärztlichen Handelns, was hier an psychiatrischen Beispielen illustriert werden sollte.

2. Ärztliche Identität hat neben der wissenschaftlichen eine entschieden ethische und gesellschaftliche Dimension. Die naive Idealisierung des Arztbildes einerseits und die blosse Anpassung an den jeweiligen Zeitgeist andererseits sind schädliche Strategien. Es braucht eine patienten- und zeitgerechte, in einem bestimmten Sinne selbstbewusste Form ärztlicher Identität für das 21. Jahrhundert.

3. Diese sicher auch in Zukunft kontroverse Debatte darf sich nicht nur in den Gesellschaftswissenschaften und der Gesundheitspolitik abspielen, sondern ist innerhalb der Ärzteschaft aktiv zu führen und weiterzuentwickeln. «Innerhalb der Ärzteschaft» heisst dabei, dass die Ausbildung der Studierenden, die Weiterbildung zum Facharzt und die kontinuierliche Fortbildung der Fachärzte in diesen Prozess eingebunden sind. Nicht im Vordergrund stehen sollten dabei gruppenspezifische Sonderinteressen etwa eines universitären Zentrums, einer Grundversorgungsklinik oder der niedergelassenen Ärzteschaft.

4. Die Grundlage jeder medizinischen Handlung bleibt die einzelne Arzt-Patienten-Beziehung. Bei allem Respekt für die unbestrittene Relevanz gesellschaftlicher und ökonomischer Rahmenbedingungen bleibt festzuhalten, dass eine gleichsam grundsätzliche Unterordnung dieses Kernelementes unter ein Primat effizienter Dienstleistung einerseits oder blosser Wunscherfüllung im Rahmen der Lifestylemedizin andererseits mit einer kohärenten und wirksamen ärztlichen Identität nicht vereinbar ist. 